\title{
Home care: more than just a visiting nurse
}

\author{
Katrina M Romagnoli, ${ }^{1}$ Steven M Handler, ${ }^{1,2,3,4,5}$ Harry Hochheiser ${ }^{1,5,6}$
}

\begin{abstract}
${ }^{1}$ Department of Biomedical Informatics, School of Medicine, University of Pittsburgh,

Pittsburgh, Pennsylvania, USA ${ }^{2}$ Division of Geriatric Medicine, Department of Medicine, University of Pittsburgh, Pittsburgh, Pennsylvania, USA ${ }^{3}$ Geriatric Research Education and Clinical Center (GRECC), Veterans Affairs Pittsburgh Healthcare System (VAPHS), Pittsburgh, Pennsylvania, USA ${ }^{4}$ Center for Health Equity Research and Promotion (CHERP), VAPHS, Pittsburgh, Pennsylvania, USA ${ }^{5}$ Geriatric Pharmaceutical Outcomes and Geroinformatics Research \& Training Program, University of Pittsburgh, Pennsylvania, USA

${ }^{6}$ Intelligent Systems Program, University of Pittsburgh Pennsylvania, USA
\end{abstract}

\section{Correspondence to} Katrina M Romagnoli, Department of Biomedical Informatics, School of Medicine, University of Pittsburgh, 5607 Baum Boulevard, BAUM 417D, Pittsburgh, PA 15206-3701, USA; kak59@pitt.edu

Received 22 July 2013 Accepted 22 July 2013 Published Online First 12 August 2013

\section{SLinked}

http://dx.doi.org/10.1136/ bmjqs-2013-002039

\footnotetext{
To cite: Romagnoli $\mathrm{KM}$ Handler SM, Hochheiser $\mathrm{H}$. BMJ Qual Saf 2013;22: 972-974.
}

When patients leave the hospital and return home with home nursing care, they go from highly supportive medical environments with potentially many physicians, nurses, aides and other professionals, to non-medical environments with formal and informal caregiver support frequently supplemented by visits from home care nurses. Patients and caregivers must struggle to absorb confusing and potentially contradictory information imparted both by multiple clinicians prior to discharge from the hospital and by home care nurses. Providers, for their part, often have incomplete understandings of home environments and patient and caregiver capabilities. Despite these difficulties, patients are largely left to themselves, expected to be engaged in their care sufficiently to own and manage their medical conditions. It is a daunting task.

Patient safety at home is as important as patient safety in hospitals. Unsafe conditions in the home can lead to unnecessary or avoidable hospitalisations. ${ }^{1}$ Home care decreases costs, improves health outcomes, and reduces hospital stays. ${ }^{2-8}$ Despite these benefits, problems exist. Around $13 \%$ of patients enrolled in home care experience an adverse event. ${ }^{9} 10$ The largest proportion of adverse events that occur among home care patients are related to medications, with 20-33\% experiencing a medication problem or adverse drug event. ${ }^{11}{ }^{12}$ Research has found that home care personnel and informal caregivers may play a role in a substantial subset of adverse events that result in hospitalisation, ${ }^{13}$ although further investigation is needed to understand the nature of the interaction. Insufficient attention to effective communication during transitional care from hospital to home may be one of the factors contributing to these patterns. ${ }^{1} 14$

Relatively little attention has been paid to the underlying causes of these adverse events and how they might be prevented.
Our literature search revealed a limited number of published manuscripts in this domain compared to other settings. To prevent hospital readmissions, improve patient outcomes and save money, more attention must be paid to home care safety.

\section{PROBLEMS IN HOME HEALTHCARE}

Home health nurses experience inadequate communication of basic patient information between the hospital, primary care, and home care after hospital discharge. ${ }^{14}$ Home care nurses receive either too much information (ie, all clinical documentation associated with an admission) or too little information (ie, just the patient's demographic information, primary discharge diagnosis and reason for the home care referral). Nurses who are not provided with actionable information must rely on patients and caregivers to share information that is hopefully relevant, appropriate, and accurate. However, patients and caregivers often cannot provide accurate information due to miscommunication, misunderstanding, and/or poor memory. ${ }^{15}$ Discharge instructions are given to all patients following a hospitalisation, but this paperwork might be lost or discarded, hard to understand, or inappropriately focused on the primary discharge diagnosis at the expense of providing information about comorbidities. ${ }^{16}$ Moreover, discharge summaries are rarely available to home care nurses. ${ }^{14}$ Reliance on patients and caregivers for vital information makes these nurses' jobs more difficult and puts patients at risk. When nurses must make decisions with incomplete or wrong information, adverse events can occur, resulting in potentially avoidable admissions/readmissions. ${ }^{1} 15$ From $5 \%$ to $79 \%$ of hospital readmissions may be avoidable. ${ }^{4}$ Improving information exchange with home healthcare would likely prevent some of those hospital stays. 
Since most care providers never enter a patient's home, they have inadequate understandings of the home care environments and the general requirements of patients, caregivers and nurses. Hospitals discharging patients, and physicians continuing to care for them postdischarge frequently do not know what the caregivers and home care nurses need to support the patient. Home care nurses usually know these things, and they would like to see improvements in care transitions, communication and interventions. ${ }^{17}$ Physicians caring for hospitalised patients are generally not in communication with home care nurses, sharing information about what happened during the hospital stay, or what was said during a follow-up visit. Conversely, home care nurses might not be able to share information about patient progress and risks with primary care physicians. Generally, none of this communication is automated or standardised, and communication of this information relies heavily on the patient and caregivers. ${ }^{14}$ There are standards available, such as the Continuity of Care Document (CCD), to share important information during care transitions. ${ }^{18}$ In the USA, home care agencies are required to use the OASIS-C document to share data. ${ }^{19}$ However, information sharing with home care nurses, patients, and caregivers is still lacking. Home care nurses would like to change this situation, but they need participation from physicians, hospitals, and healthcare systems. Better data might help convince these stakeholders of the need for change.

Blais et $a l^{20}$ provide evidence that argues for action. In a large, national, retrospective study of Canadian home care adverse events, they found an overall adverse event rate of $4.2 \%$, with $56 \%$ of events deemed preventable. Falls, wound infections, psychosocial, behavioural or mental health problems, and medication errors were among the most frequent causes of adverse events. Over $90 \%$ of these adverse events were associated with higher use of health care resources, $68.8 \%$ with disability and $7.5 \%$ with death. They also found that patients contribute to $48.4 \%$ of adverse events, caregivers contribute to $20.4 \%$, and health care personal contribute to $46.2 \%$.

These results support our argument that better communication among clinicians, caregivers and patients can avoid adverse events and improve the safety of home care. In our recent study, home care nurses identified a number of frequent, high-impact information and communication needs experienced by patients that have received minimal attention in the literature. ${ }^{14}$ These include information about medication regimens, details about the severity of the patient's condition, information about hospital discharge, non-medication care regimens such as wound care or home safety, the extent of necessary care at home, and which providers are best suited for that care. For instance, nurses told us that patients and caregivers often do not know how to properly care for wounds, follow medication regimens correctly, manage durable medical equipment, or keep their houses free of hazards. These are just a few examples of how patient and caregiver actions can lead to problems at home. Improved communication and education about these high-risk problems for patients could address the most dangerous home care safety issues.

\section{POSSIBLE SOLUTIONS}

Healthcare systems, clinicians, and researchers need to better engage home care nurses to understand their workflow, their information needs, their patients, and their work environments. In our experience, home care nurses are willing and enthusiastic participants who are eager to improve their patients' care. Researchers will find this a ripe field of study, particularly in terms of the development of new interventions designed to improve the quality and safety of healthcare that patients receive in the home through better patient engagement strategies. For instance, hospitals often have standard discharge instructions that are provided to the patient and perhaps to the primary care physician that summarise what happened in the hospital, new treatments, or medications and other important information. Project RED (the REengineered Discharge program) provides patients and their physicians with individualised instruction booklets and other materials at discharge. ${ }^{21}$ These interventions were associated with a decrease in the rate of hospital readmission. Including home care nurses in that information exchange, where applicable, is a simple intervention. Another possible solution involves the development of improved protocols for reporting adverse events that occur in the home, and using this information to determine better prevention strategies. ${ }^{20}$ If adverse events are poorly recorded and monitored, little hope exists in learning the patterns of these events in the home, let alone preventing them in the future. Finally, home care providers, hospitals and physicians need to engage caregivers at a greater rate. Sick, elderly patients are likely to rely heavily on caregivers, who often contribute to adverse events because of lack of appropriate information. ${ }^{13}$ Developing interventions to improve caregiver communication and training in the proper care of the patient might minimise the risk caregivers introduce. Other solutions include telehealth interventions, such as phone calls to the patient by the home care nurse between visits, ${ }^{21} 22$ remote physiologic monitoring, medication delivery units, and information portals providing patients, caregivers, and clinicians with consistent information.

Improvements in information sharing and communication among all clinicians and caregivers will not solve all problems in home care, nor prevent all adverse events. However, some of these changes are relatively inexpensive and easy to implement, and ought to be considered by researchers and health systems as an initial intervention. Home care nurses provide a valuable service to their patients by helping 
them stay healthier, reducing costs, and helping them avoid hospitals and long-term care facilities. ${ }^{2-8}$ Approaches that do not consider the input of these critical yet underappreciated healthcare providers run the risk of missing valuable opportunities to reduce adverse events and improve care received at home. The experience and expertise of home care nurses have the potential to guide substantial improvements in patient safety and care at a relatively low cost. Ignoring them is not just foolish, but dangerous.

Funding This work was supported by the NLM Fellowship 5 T15 LM007059-24, Agency for Healthcare Research and Quality (R01HS018721) and National Institute of Aging grants (R01AG027017; P30AG024827; K07AG033174).

\section{Competing interests None.}

Contributors All authors contributed equally to this paper.

Provenance and peer review This was an invited editorial; internally peer reviewed.

\section{REFERENCES}

1 Coleman EA. Falling through the cracks: challenges and opportunities for improving transitional care for persons with continuous complex care needs. J Am Geriatr Soc 2003;51:549-55.

2 Mamolen NL, Brenner PS. The impact of a burn wound education program and implementation of a clinical pathway on patient outcomes. J Burn Care Rehabil 2000;21:440-5; discussion 439.

3 Leff B, Burton L, Mader SL, et al. Comparison of functional outcomes associated with hospital at home care and traditional acute hospital care. J Am Geriatr Soc 2009;57:273-8.

4 Hughes SL, Ulasevich A, Weaver FM, et al. Impact of home care on hospital days: a meta analysis. Health Serv Res 1997;32:415-32.

5 Elkan R, Kendrick D, Dewey M, et al. Effectiveness of home based support for older people: systematic review and meta-analysis. BMJ 2001;323:719-25.

6 Counsell SR, Callahan CM, Clark DO, et al. Geriatric care management for low-income seniors: a randomized controlled trial. JAMA 2007;298:2623-33.

7 Beales JL, Edes T. Veteran's Affairs Home Based Primary Care. Clin Geriatr Med 2009;25:149-54, viii-ix.

8 Barrett DL, Secic M, Borowske D. The Gatekeeper Program: proactive identification and case management of at-risk older adults prevents nursing home placement, saving healthcare dollars program evaluation. Home Healthc Nurse 2010;28:191-7.

9 Madigan EA. A description of adverse events in home healthcare. Home Healthc Nurse 2007;25:191-7.

10 Sears N, Baker GR, Barnsley J, et al. The incidence of adverse events among home care patients. Int J Qual Health Care 2013;25:16-28.

11 Gray SL, Mahoney JE, Blough DK. Adverse drug events in elderly patients receiving home health services following hospital discharge. Ann Pharmacother 1999;33:1147-53.

12 Meredith S, Feldman PH, Frey D, et al. Possible medication errors in home healthcare patients. J Am Geriatr Soc 2001;49:719-24.

13 Johnson KG. Adverse events among Winnipeg Home Care clients. Healthc Q 2006;9, Special Issue:127-34.

14 Romagnoli KM, Handler SM, Ligons FM, et al. Home-care nurses' perceptions of unmet information needs and communication difficulties of older patients in the immediate post-hospital discharge period. BMJ Qual Saf 2013;22: 324-32.

15 Van Walraven C, Bennett C, Jennings A, et al. Proportion of hospital readmissions deemed avoidable: a systematic review. CMAJ 2011;183:E391-402.

16 Fagermoen MS, Hamilton G. Patient information at dischargea study of a combined approach. Patient Educ Couns 2006;63:169-76.

17 Smith SB, Alexander JW. Nursing perception of patient transitions from hospitals to home with home health. Prof Case Manag 2012;17:175-85.

18 Murphy LS, Wilson ML, Newhouse RP. Improving care transitions through meaningful use stage 2: continuity of care document. J Nurs Adm 2013;43:62-5.

19 Ferranti JM, Musser RC, Kawamoto K, et al. The clinical document architecture and the continuity of care record: a critical analysis. J Am Med Inform Assoc 2006;13: 245-52.

20 Blais R, Sears N, Doran D, et al. Assessing adverse events among home care clients in three Canadian provinces using chart review. BMJ Qual Saf 2013;22:989-97.

21 Jack BW, Chetty VK, Anthony D, et al. A reengineered hospital discharge program to decrease rehospitalization: a randomized trial. Ann Intern Med 2009;150:178-87.

22 Coleman EA, Parry C, Chalmers S, et al. The care transitions intervention: results of a randomized controlled trial. Arch Intern Med 2006;166:1822-8. 